\title{
Qualitative evaluation of paediatric burn injury in Malawi: assessing opportunities for injury prevention
}

\author{
Marissa Bane', Ruth Kaima ${ }^{2}$, Suzgo Mapala ${ }^{3}$, Bruce Cairns ${ }^{4}$ and \\ Anthony Charles ${ }^{5}$
}

\begin{abstract}
Introduction: The burden of burn injury in sub-Saharan Africa is high and children bare a disproportional share of the injury burden.

Methods: This is a prospective qualitative study of paediatric burn survivors (age, $\leq \mathbf{8}$ years) admitted to Kamuzu Central Hospital $(\mathrm{KCH})$ in Lilongwe, Malawi.

Results: There were a total of 72 guardians interviewed for the purpose of the study. The most frequent mechanisms of burn injury were flames and scalds in $44.4 \%$ and $38.9 \%$, respectively. Mothers were present at the time of the burn injury in only $23.6 \%$ of cases, while $22.2 \%$ of children were unmonitored at the time of injury. A total of $55.6 \%$ of burn injuries was cooking-related.

Conclusion: Burn prevention strategies are necessary for addressing the high rates of burns for children in sub-Saharan Africa. The must be focused on mothers and caregivers burn education in the home, particularly as it relates to cooking and parental oversight.
\end{abstract}

\section{Keywords}

Burn injury in children, Malawi, burn prevention, burns in sub-Saharan Africa

\section{Introduction}

Burns are a significant burden of paediatric injuries. Mortality from paediatric burns is most pronounced in low- and middle-income countries (LMICs) where over $90 \%$ of burn-related paediatric deaths occur. This is equivalent to 3.4 burn-related deaths per 100,000 children in LMICs compared to 0.5 deaths per 100,000 in high-income countries. By region, the World Health Organization's (WHO) African region has the highest rate of paediatric burn-related deaths at 7.03 per 100,000 children. ${ }^{1}$

There are very few acute burn units in sub-Saharan Africa and currently most are focused on the treatment of burn injury, specifically wound care, and less so on exploring the environmental and behavioural issues surrounding the injury event. Due to the high

\footnotetext{
'Undergraduate student, Gillings School of Global Public Health, University of North Carolina at Chapel Hill, North Carolina, USA ${ }^{2}$ Student and data clerk, UNC-Project Malawi, Lilongwe, Malawi ${ }^{3}$ Burn Surgeon, Department of Surgery, University of North Carolina at Chapel Hill, North Carolina, USA

${ }^{4}$ Associate Professor of Surgery, Department of Surgery, University of North Carolina at Chapel Hill, North Carolina, USA

${ }^{5}$ Associate Professor of Surgery, Kamuzu Central Hospital, Lilongwe, Malawi; Department of Surgery, University of North Carolina at Chapel Hill, North Carolina, USA
}

\section{Corresponding author:}

Anthony Charles, Associate Professor of Surgery, UNC School of Medicine, Adjunct Associate Professor of Public Health, Gillings School of Global Public Health, University of North Carolina, 4008 Burnett Womack Building, CB 7228, Chapel Hill, NC 27599, USA. Email: anthchar@med.unc.edu 
morbidity and mortality associated with burn injury, we sought to better understand the circumstances surrounding the burn injury, so as to inform burn injury prevention strategies.

\section{Methods}

This study is a prospective qualitative survey and analysis based at the 31-bed Burn Unit at Kamuzu Central Hospital $(\mathrm{KCH})$, Malawi. $\mathrm{KCH}$ serves as a referral centre for approximately five million people in the central region of Malawi.

A 35-question quantitative survey in the local language, Chichewa, addressed burn aetiology, circumstances and environment in children aged $\leq 8$ years. The survey consisted of five main topic domains: patient demographics; socio-demographic characteristics of primary guardian; home cooking style; child care arrangement; and burn event information. A data clerk administered the survey at $\mathrm{KCH}$. Data were collected directly from each patient's primary guardian.

\section{Results}

\section{Patient demographics}

We collected data on 72 patients. The average patient age was $3 \pm 2$ years, and $41(57 \%)$ patients were boys. Forty $(55.6 \%)$ patients resided in high-density areas, and $16(22.2 \%)$ patients were transferred from another hospital.

\section{Guardian socio-demographics}

Seventy-two primary guardians for interviewed. All of the primary guardians were women, and $64(88.9 \%)$ were the patients' mothers. Due to their employment status, $44(61 \%)$ guardians reported being at home with their children every day.

The number of people in each household was in the range of 2-4 per household in $35(48.61 \%)$ cases, while 30 guardians $(41.7 \%)$ reported $5-7$ people in their household. Overcrowding in the house did not seem to be an area of concern for most of the guardians.

\section{Cooking practices}

Forty-two $(58.3 \%)$ guardians used charcoal and 27 guardians $(37.5 \%)$ used wood, while only three $(4.2 \%)$ guardians used electricity for cooking. Fifty-five (76.4\%) guardians cooked at floor level, 14 (19.4\%) guardians cooked on a low stove, and three $(4.2 \%)$ guardians cooked on a high stove. Forty $(55.6 \%)$ and $27(37.5 \%)$ guardians cooked outdoors and indoors, respectively, and five $(6.9 \%)$ guardians varied cooking location depending on weather conditions.

\section{Child care characteristics of patient}

During daytime hours, $30(41.7 \%)$ mothers were responsible for looking after their children. Ten $(13.9 \%)$, grandmothers watched over the children, and six $(8.3 \%)$ sisters watched over the children. During this time, 37 (51.4\%) of those responsible for the children were taking care of one or two children, and $25(34.7 \%)$ were responsible for three or four children.

\section{Burn incident characteristics}

Thirty-two (44.4\%) patients sustained burn injury by open flame fire, $28(38.9 \%)$ patients by hot water, and eight $(11.1 \%)$ patients by hot porridge resulting in scalds. Twenty-eight $(38.9 \%)$ patients were burned in the late afternoon, and $16(22.2 \%)$ sustained their burn injury in the early morning. Forty $(55.6 \%)$ patients sustained their injury outdoors.

Forty $(55.6 \%)$ patients had cooking-related burns. A total of $97.5 \%$ of cooking-related burns took place in the normal cooking area. Twelve $(37.5 \%)$ burns not due to cooking were related to heating; furthermore, six $(18.8 \%)$ burn injuries were each related to bathing water and the use of candles or open flames as a light source, respectively. At the time of the burn, 17 (23.6\%) mothers were with their burn-injured child, and 16 $(22.2 \%)$ children were unmonitored or unsupervised at the time of the burn injury. Nine $(12.5 \%)$ patients were being taken care of by a sibling, eight $(11.1 \%)$ by a family friend, and five $(6.9 \%)$ by a grandmother.

\section{Discussion}

This study describes the socio-demographics of our burn patients and their guardians and the circumstances surrounding the burn injury event in patients presenting to a hospital in sub-Saharan Africa. Given the significant burden of paediatric burn injuries, prevention is essential to reduce burn-related mortality and morbidity. Prevention strategies need to be tailored to the specific environment taking into account local risk factors, specific aetiologic patterns, available resources and target high-risk populations. ${ }^{2,3}$

Surgical diseases have largely been ignored in the global health sphere up until recently, and addressing injury, the greatest driver of global surgical disease burden is imperative. ${ }^{4}$ Burn injury care and prevention have been regarded as too expensive to deliver amid competing healthcare priorities. Furthermore, health policy in sub-Saharan Africa has not been informed 
by good research. All these factors have led to the relative inertia in the implementation of national injury prevention initiatives.

In this study, the majority of burn injuries occurred at home and were cooking-related. Food preparation using open cast iron pots constitute a major hazard for small children and toddlers as they often play around the cooking area; moreover, most cooking in subSaharan Africa is ground level cooking, which is accessible to children. ${ }^{5}$ Burn prevention strategies should target mothers and caregivers at home. Many of our burn patients were unsupervised at the time of injury. Prevention programs targeted at women, that is focused on safe cooking practices such as never leaving food unattended on the stove or securing the area around open flames will help mitigate burn injury incidence and its sequelae, particularly in the paediatric population. ${ }^{6}$ Furthermore, an educational program that emphasises childhood supervision at all times is essential. In addition, the embrace of initiatives such as the World Food Program Stove Initiative shows that stoves may not only reduce burn injury but also attenuate lung injury due to smoke exposure in women and children. ${ }^{7}$ In addition, initiatives that promote the construction of communal kitchens equipped with a safe fuel-efficient stove at chest level with a controlled entrance should be encouraged. This will require significant community education. ${ }^{8}$

This study had several limitations. We have a relatively small sample and we only interviewed patients admitted to $\mathrm{KCH}$ who typically sustained more serious burn injury, exposing our findings to selection bias. In addition to participant willingness, personality traits, injury characteristics, support systems and other demographic variables all influence the fidelity of the responses given. Our findings may not be representative of all of Africa, but we believe it is representative of the region.

\section{Conclusion}

This qualitative analysis highlights the sociodemographic and behavioural determinants of burn injury. A multi-targeted strategy for burn injury prevention focusing on developing programs for caregiver education and cooking practices is imperative.

\section{Acknowledgments}

The authors thank NC Jaycee Burn Center for providing administrative assistance throughout the research process.

\section{Declaration of conflicting interests}

The author(s) declared no potential conflicts of interest with respect to the research, authorship, and/or publication of this article.

\section{Funding}

The author(s) disclosed receipt of the following financial support for the research, authorship, and/or publication of this article: All funding for the study was given through the Honors Carolina Burch Fellowship. The University of North Carolina Project in Lilongwe, Malawi, and the UNC Department of Surgery.

\section{References}

1. World Health Organization. Deaths estimates for 2008 by cause for WHO Member States. Geneva: World Health Organization, 2011.

2. Atiyeh BS, Costagliola M and Hayek SN. Burn prevention mechanisms and outcomes: pitfalls, failures and successes. Burns 2009; 35: 181-193.

3. Stone DH. Research on injury prevention: time for an international agenda. Epidemiol Commun Health 1996; 50: $127-130$

4. Raykar NP, Yorlets RR, Liu C, Greenberg SL, Kotagal $\mathrm{M}$, Goldman R, et al. A qualitative study exploring contextual challenges to surgical care provision in 21 LMICs. Lancet 2015; 385: S15.

5. Onuba $\mathrm{O}$ and Udoidoik E. The problem and prevention of burns in developing countries. Burns 1987; 3(5): 382-385.

6. WFP Stove Initiative To Help Protect Women And Environment. Available at: https://www.wfp.org/stories/ wfp-launches-safe-stoves-initiative (accessed 16 September 2015).

7. Parbhoo A, Louw QA and Grimmer-Somers K. Burn prevention programs for children in developing countries require urgent attention: a targeted literature review. Burns 2010; 36: 164-175.

8. Lewis JJ, Bhojvaid V, Brooks N, Das I, Jeuland MA, Patange $\mathrm{O}$, et al. Piloting improved cookstoves in India. J Health Commun 2015; 20(Suppl. 1): 28-42. 